\title{
The application of localized surface plasmons resonance in Ag nanoparticles assisted Si chemical etching
}

Ruiqiang Ding, Han Dai, Meicheng Li, Jinjer Huang, Yingfeng Li, Mwenya Trevor, and Kevin P. Musselman

Citation: Appl. Phys. Lett. 104, 011602 (2014); doi: 10.1063/1.4855615

View online: https://doi.org/10.1063/1.4855615

View Table of Contents: http://aip.scitation.org/toc/apl/104/1

Published by the American Institute of Physics

\section{Articles you may be interested in}

High aspect ratio silicon etch: A review

Journal of Applied Physics 108, 051101 (2010); 10.1063/1.3474652

Enhanced emission from Si-based light-emitting diodes using surface plasmons

Applied Physics Letters 88, 161102 (2006); 10.1063/1.2195695

A role of illumination during etching to porous silicon oxidation

Applied Physics Letters 75, 826 (1999); 10.1063/1.124526

Solid state photovoltaic cells based on localized surface plasmon-induced charge separation

Applied Physics Letters 99, 182110 (2011); 10.1063/1.3659476

Layer roughness reduction and light harvest from Ag nanowires on a silicon surface through wet etching embedding

Applied Physics Letters 111, 021601 (2017); 10.1063/1.4993758

Enhanced localized surface plasmon resonance obtained in two step etched silicon nanowires decorated with silver nanoparticles

Applied Physics Letters 103, 143124 (2013); 10.1063/1.4824646

This article may be downloaded for personal use only. Any other use requires prior permission of the author and AIP Publishing. The following article appeared in Ding, R., Dai, H., Li, M., Huang, J., Li, Y., Trevor, M., \& Musselman, K. P. (2014). The application of localized surface plasmons resonance in Ag nanoparticles assisted Si chemical etching. Applied Physics Letters, 104(1), 011602 and may be found at https://doi.org/10.1063/1.4855615

\section{PHYSICS TODAY}

MANAGER'S GUIDE

Accelerate R\&D with Multiphysics Simulation

\section{READ NOW}

PRESENTED BY

$\checkmark \subset \bigcirc M S O L$ 


\title{
The application of localized surface plasmons resonance in $\mathrm{Ag}$ nanoparticles assisted Si chemical etching
}

\author{
Ruiqiang Ding, ${ }^{1, a)}$ Han Dai, ${ }^{1, a)}$ Meicheng Li, ${ }^{1,2, b)}$ Jinjer Huang, ${ }^{3}$ Yingfeng Li, ${ }^{1}$ \\ Mwenya Trevor, ${ }^{1}$ and Kevin P. Musselman ${ }^{4}$ \\ ${ }^{1}$ State Key Laboratory of Alternate Electrical Power System with Renewable Energy Sources, \\ School of Renewable Energy, North China Electric Power University, Beijing 102206, China \\ ${ }^{2}$ Suzhou Institute, North China Electric Power University, Suzhou 215123, China \\ ${ }^{3}$ Harbin University of Science and Technology, Harbin 150006, China \\ ${ }^{4}$ Department of Physics, University of Cambridge, Cambridge CB3 OHE, United Kingdom
}

(Received 4 March 2013; accepted 8 December 2013; published online 9 January 2014)

\begin{abstract}
Localized surface plasmons excited by Ag nanoparticles are introduced in the chemical etching process of silicon. A special crateriform structure with gradually varying radius is achieved by the surface electromagnetic field enhancement effect of localized surface plasmons resonance (LSPR). Theoretical analysis demonstrates that the formation kinetics of the crateriform structures conforms to the local electromagnetic field enhancement and forward scattering induced by LSPR. The LSPR assisted photocatalytic etching offers a potential approach for the preparation of the surface microstructures used in optoelectronic devices. (C) 2014 AIP Publishing LLC.
\end{abstract}

[http://dx.doi.org/10.1063/1.4855615]

Localized surface plasmons (LSP) of Ag nanoparticles have recently attracted a lot of attention in many fields, due to their strong localized electromagnetic field enhancement and light scattering properties. ${ }^{1}$ Examples of such fields include Raman scattering, ${ }^{2}$ photovoltaic, ${ }^{3}$ and sensors. ${ }^{4}$ Recently, the LSP, especially localized surface plasmons resonance (LSPR) of noble metal nanoparticles, can dramatically amplify the localized electromagnetic fields and, therefore, have become an interesting topic in the visiblelight photocatalyst process. ${ }^{5,6}$

Silicon chemical etching is a simple and effective method to fabricate surface microstructures, and can be influenced by the electromagnetic field. ${ }^{7,8}$ By varying the electromagnetic field intensity, round, square, and triangular shaped surface morphologies have been obtained in Si chemical etched process. ${ }^{9,10}$ However, the morphologies of the etched structure by this method are finite. ${ }^{11}$ Therefore, it is necessary and important to enrich the Si chemical etching method in the fabrication of microstructures. Here, we introduce the effect of LSPR on $\mathrm{Ag}$ nanoparticles assisted $\mathrm{Si}$ chemical etching process.

In this Letter, the effect of LSPR on the Si etching process, assisted by Ag nanoparticles under laser irradiation, is investigated. The photocatalytic mechanism of LSPR on the morphology of the etched structure is proved by analyzing the localized electromagnetic field enhancement and electron transport by Mie and electrochemistry theories. The obtained crateriform structure could enhance the light collection and light emission properties, and can be used in light emitting diodes (LEDs) and solar cells. This work shows an efficient way of fabricating the material surface microstructure.

In experimental, a krypton ion laser is tuned to a short wavelength $(\lambda=476 \mathrm{~nm})$ to excite LSPR on $\mathrm{Ag}$

\footnotetext{
${ }^{\text {a) }}$. Ding and H. Dai contributed equally to this work.

${ }^{b)}$ Author to whom correspondence should be addressed. Electronic mail: mcli@ncepu.edu.cn. Fax: +86 86172951.
}

nanoparticles, which possesses the properties of high light intensity, monochromaticity, and parallelism. ${ }^{11-13}$ P-type $\mathrm{Si}(111)$ wafers $(1-2 \Omega \cdot \mathrm{cm})$ that are polished on one side are used, and by sputtering deposition and annealing processes, $\mathrm{Ag}$ nanoparticles are deposited on silicon surface. Then, the samples are etched with laser illumination/irradiation for $60 \mathrm{~s}$ in $\mathrm{HF} / \mathrm{H}_{2} \mathrm{O}_{2}$ etchant (volume ratio; $\mathrm{HF}: \mathrm{H}_{2} \mathrm{O}_{2}: \mathrm{H}_{2} \mathrm{O}$ $=1: 5: 10$ ). Every sample has two regions for comparison, one formed with laser illumination and the other without.

In the laser illuminated region, the crateriform structures are formed on Si surface as shown in Fig. 1(a), and the region without illumination only exhibit ordinary straight holes (Figs. 1(b) and 1(c)), which is typical of porous $\mathrm{Si}$ structure etching assisted by $\mathrm{Ag}$ nanoparticles. ${ }^{14}$ In both regions, the $\mathrm{Ag}$ nanoparticles are left at the bottom of etched structure after the etching process. In the illuminated region, the special structure characteristics of etched morphology are shown in the inset image of Fig. 1(a), which shows a crateriform top and a cone-shaped bottom. The diameter of crateriform tops range from $60 \mathrm{~nm}$ to $120 \mathrm{~nm}$, and the corresponding $\mathrm{Ag}$ nanoparticles have a relative diameter ranging from $20 \mathrm{~nm}$ to $50 \mathrm{~nm}$. The cone does not have obvious orientation, and the diameter of each crateriform structure corresponds to the $\mathrm{Ag}$ nanoparticles. Obviously, the two parts of the etching structure exhibit significant differences in morphology, which indicates that two different etching mechanisms work in the etching process.

From the experiment results, laser illumination in the etching process has an influence on the morphologies of the special crateriform structure. As we know, the morphology of etching structure can be greatly affected by the change of photogeneration carriers in the $\mathrm{Si}$ substrate under illumination. ${ }^{10}$ It is worth noting that the intensity of the electromagnetic field near $\mathrm{Ag}$ nanoparticles can be greatly enhanced by the excitation of LSPR, which can have great influence on the photogeneration carriers in the Si substrate. ${ }^{15,16}$ In our work, the formation of the crateriform structure surrounding 


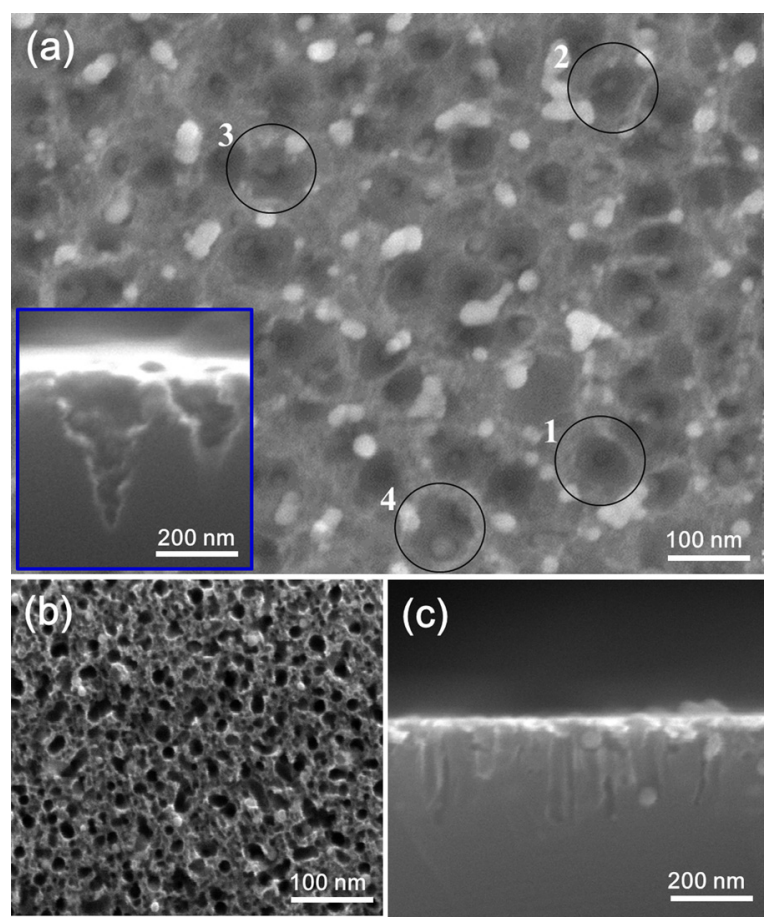

FIG. 1. SEM images of Si etch topography; (a) Plane-view of crateriform Si microstructure in laser illuminated scale with an inset image for crosssectional view. Plane-view (b) and Cross-sectional view (c) of the same sample without light illumination, separately.

Ag nanoparticles indicates that LSPR may have an influence on the morphologies of the special structure. Thus, Mie theory, for its accurate description of the electromagnetic field of metal nanoparticles, is applied to study the electromagnetic field distribution around Ag nanoparticles. ${ }^{9}$ From previous studies, the electromagnetic field enhancement region of $\mathrm{Ag}$ nanoparticles can be described by the scattering cross section (Qsca), and the expression of Qsca is shown in Eq. (1) below

$$
Q_{s c a}=\frac{2}{x^{2}} \sum_{n=1}^{\infty}(2 n+1)\left(\left|a_{n}\right|^{2}+\left|b_{n}\right|^{2}\right),
$$

where $a_{n}$ and $b_{n}$ are the scattering coefficients of the $n$th scattering electromagnetic mode. $x=2 \pi N a / \lambda$, where $a$ is the particle diameter and $\lambda$ is the wavelength of the incidence light. Taking into account the effect of the interface between the etchant and Si surface, the effective permittivity of $N$ is set as the average value of Si substrate and etchant. The Qsca of Ag nanoparticles with different diameters at wavelengths ranging from $300 \mathrm{~nm}$ to $1100 \mathrm{~nm}$ is calculated according to Eq. (1), as shown in Fig. 2. Under the laser illumination with a wavelength of $476 \mathrm{~nm}$, the values of Qsca increase with the diameter of Ag nanoparticles. It is surprising that the Qsca of $\mathrm{Ag}$ nanoparticles with diameters ranging from $35 \mathrm{~nm}$ to $50 \mathrm{~nm}$ are approximately the same as the diameters of the crateriform structures, as shown in Table I. Both the Qsca and the diameter of crateriform structure are increasing in accordance to the increase of the nanoparticle diameter.

Fig. 3 shows a schematic which depicts the etching process and is presented for better illustration of the similarity in value of the Qsca and the crateriform. Under illumination, the scattering of $\mathrm{Ag}$ nanoparticle is shown in the schematic

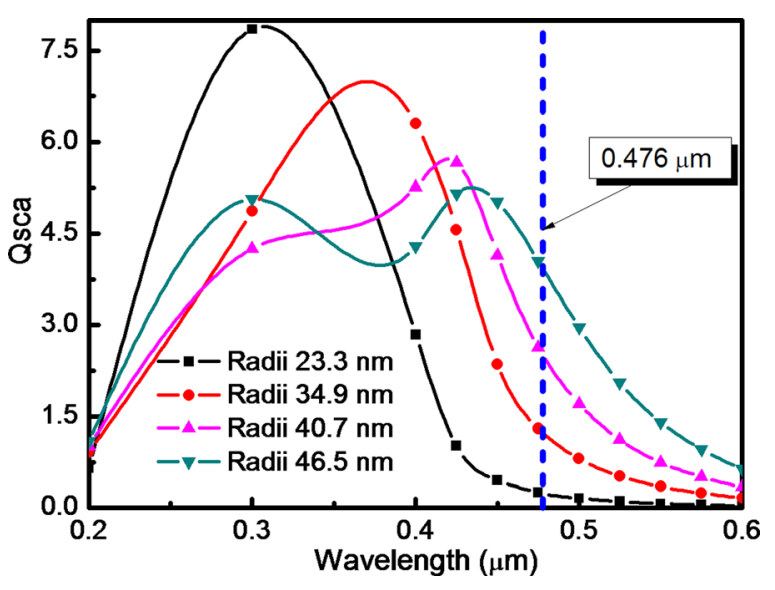

FIG. 2. Scattering cross sections of Ag nanoparticles with different particle diameters.

diagram and the main scattering direction is forward scattering. In the vicinity of the Ag nanoparticle, the electromagnetic field on the silicon substrate can be greatly amplified. ${ }^{15,16}$ A physical way to visualize the enhancement of the electromagnetic field is to consider the particle as having localized the plane wave of the light as a dipole field surrounding the sphere. The incident laser field surrounding the particle is described as follows: ${ }^{17}$

$$
\left.E_{\text {induced }}=\left[\left(\varepsilon_{1}(\omega)-\varepsilon_{2}\right) /\left(\varepsilon_{1}(\omega)+2 \varepsilon_{2}\right)\right]\right] E_{\text {laser }} .
$$

The electromagnetic field induced at the surface of the sphere is related to the laser field by Eq. (2), where $\varepsilon_{1}(\omega)$ is complex, the frequency-dependent dielectric function of the metal and $\varepsilon_{2}$ is the relative permittivity of the ambient medium. At the LSPR frequency, $\operatorname{Re}\left(\varepsilon_{1}\right)+2 \varepsilon_{2}=0$, which means that the $\mathrm{Ag}$ nanoparticles act as an antenna which amplifies the intensity of the scattered electromagnetic field and the overall enhancement of magnitude is roughly two orders. Due to this enhancement and the forward scattering of the electromagnetic field in the Qsca region, a lot of photogenerated carriers are concentrated on the side walls of etched structure as shown in Fig. 3. The direct dissolution of $\mathrm{Si}$ on the side walls caused by the photogenerated carriers can be described by the chemical reaction below

$$
\mathrm{Si}+n h^{+}+6 H F=n H^{+}+H_{2} \mathrm{SiF}_{6}+((4-n) / 2) H_{2} \uparrow,
$$

where $\mathrm{n}$ is the number of holes per dissolved $\mathrm{Si}$ atom, and $\mathrm{h}^{+}$ stands for photogenerated carriers in the silicon substrate. A lot of photogenerated carriers greatly accelerate the etching process. Thus, the etching rate is much higher than that of

TABLE I. The diameters of crateriform structures, Ag nanoparticles, and simulated scattering cross sections Qsca (the numbers indicate the sampled crateriform structures in Fig. 1(a)).

\begin{tabular}{lcrc}
\hline \hline Number & $\begin{array}{c}\text { Crater } \\
\text { diameter }(\mathrm{nm})\end{array}$ & $\begin{array}{c}\text { Qsca } \\
(\mathrm{nm})\end{array}$ & $\begin{array}{c}\text { Ag nanoparticles } \\
\text { diameter }(\mathrm{nm})\end{array}$ \\
\hline 1 & 69.8 & 5.8 & 23.3 \\
2 & 87.2 & 45.2 & 34.9 \\
3 & 116.3 & 107.2 & 40.7 \\
4 & 110.5 & 188.3 & 46.5 \\
\hline \hline
\end{tabular}




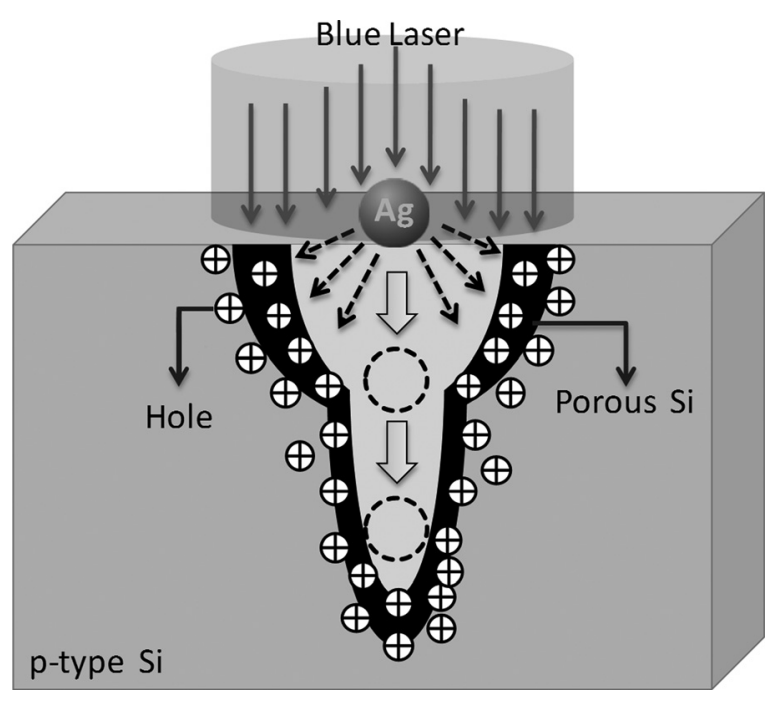

FIG. 3. Schematics of the formation mechanism of crateriform structure with laser illumination. The dashed arrows represent light scattered by Ag nanoparticle, and the dashed circles depict the sink process of Ag nanoparticle in the etching process.

the region without illumination, which results in similar value of the Qsca and the crateriform. Meanwhile, the intensity of electromagnetic field is not uniform in the Qsca and it reduces as the distance from the metal nanoparticles increases. ${ }^{18}$ Thus, in the Qsca region, the etching rate gradually decreases as the distance from $\mathrm{Ag}$ nanoparticles increases, which forms the special crateriform morphology. Previous studies have shown that the cone-shaped bottom formed under the crateriform top, as depicted in Fig. 3, exhibits typical Ag nanoparticles assisted Si etching results. ${ }^{7}$ This indicates that the LSPR only works at a certain depth near the surface of Si and is caused by the significant reduction in electromagnetic field intensity. This decrease can be attributed to two aspects: The electromagnetic field absorption of porous $\mathrm{Si}$ on the side walls of the etched structure as shown in Fig. 1(a); and the shallow penetration of light in silicon (about $450 \mathrm{~nm}$ ) at the wavelength of $476 \mathrm{~nm} .{ }^{19}$ Therefore, from the above analysis, it has been proven that LSPR is effective in the formation of the crateriform structure in etching process.

However, in some instances, the Qsca and the etching diameters of some $\mathrm{Ag}$ nanoparticles do not comply with the above rules. For Ag nanoparticles with diameter less than $30 \mathrm{~nm}$, the Qsca is much smaller than the diameter of particle, indicating that the Mie scattering theory is not effective. In this situation, the etching process can be explained by the Ag nanoparticles assisted electrochemical catalytic etching. $\mathrm{Ag}$ nanoparticles are oxidized by $\mathrm{H}_{2} \mathrm{O}_{2}$, and the holes are generated on the $\mathrm{Ag}$ nanoparticles. Then, the holes are injected into the valence band of $\mathrm{Si}$, which leads to the local oxidation and dissolution of Si atoms underneath the Ag particles. $^{20}$ By preliminary calculation, the turnover frequency is about $3500 / \mathrm{min}$ at common $\mathrm{Ag}$ nanoparticles assisted $\mathrm{Si}$ etching and increased to around 5000/min under illumination. Under laser illumination, to some extent, the catalytic activity of Ag nanoparticles has increased. Thus, the abnormally large diameters of the etching morphology of these small particles can be attributed to the high electrochemical catalytic activity of smaller Ag nanoparticles.

It is well known that a textured surface with coneshaped holes can significantly improve the efficiency of the LED and the solar cells. ${ }^{21}$ This crateriform structure has both light trapping and light collecting properties and has great potential to be applied in the devices.

In summary, LSPR has been employed for Si surface microstructure fabrication in chemical etching process. The crateriform structure is formed on silicon surface in the etching process. The mechanism of the formation of the crateriform structure is revealed by Mie theory. The calculated results prove that LSPR has a dominant role in the formation of the crateriform structure. Furthermore, the results also indicate that LSPR only works at a depth near the surface of silicon in the etching process. This work shows an efficient way for application of LSPR in the chemical etching process to enrich the morphologies of the material surface microstructure.

This work was supported by the National Natural Science Foundation of China (Nos. 91333122, 51372082, 51172069, 50972032, 61204064, and 51202067), and Ph.D. Programs Foundation of Ministry of Education of China (20130036110012, 20110036110006), and the Fundamental Research Funds for the Central Universities (Key Project No. 11ZG02).

${ }^{1}$ W. L. Barnes, A. Dereux, and T. W. Ebbesen, Nature 424, 824 (2003).

${ }^{2}$ K. Saravanan, B. K. Panigrahi, R. Krishnan, and K. G. M. Nair, J. Appl. Phys. 113, 033512 (2013).

${ }^{3}$ T. Holmgaarda and S. I. Bozhevolnyi, Appl. Phys. Lett. 92, 011124 (2008).

${ }^{4}$ A. J. Haes, S. L. Zou, G. C. Schatz, and R. P. Van Duyne, J. Phys. Chem. B 108, 109 (2004).

${ }^{5}$ P. Wang, B. B. Huang, X. Y. Qin, and X. Y. Zhang, Angew. Chem. Int. Ed. 47, 7931 (2008).

${ }^{6}$ P. Wang, B. B. Huang, X. X. Zhang, and X. Y. Qin, Chem. Eur. J. 15, 1821 (2009).

${ }^{7}$ E. A. Ponomarev and C. Lévy-Clément, Electrochem. Solid-State Lett. 1, 42 (1998).

${ }^{8}$ J. Salonen, V.-P. Lehto, M. Bjökqvist, and E. Laine, Appl. Phys. Lett. 75, 826 (1999).

${ }^{9}$ C. F. D. Bohren and R. Huffman, Absorption and Scattering of Light by Small Particles (Wiley, New York, 1983).

${ }^{10}$ C. Lévy-Clément, A. Lagoubi, and M. Tamkiewicz, J. Electrochem. Soc. 141, 958 (1994).

${ }^{11}$ S. Pillai, K. R. Catchpole, T. Trupke, G. Zhang, J. Zhao, and M. A. Green, Appl. Phys. Lett. 88, 161102 (2006).

${ }^{12}$ S. Chattopadhyay, X. L. Li, and P. W. Bohn, J. Appl. Phys. 91, 6134 (2002).

${ }^{13}$ X. H. Xiao, F. Ren, X. D. Zhou, T. C. Peng, W. Wu, X. N. Peng, X. F. Yu, and C. Z. Jiang, Appl. Phys. Lett. 97, 071909 (2010).

${ }^{14}$ F. Wang, H. Y. Yu, X. C. Wang, J. S. Li, X. W. Sun, M. F. Yang, S. M. Wong, and H. Y. Zheng, J. Appl. Phys. 108, 024301 (2010).

${ }^{15}$ H. Dai, M. C. Li, and Y. F. Li, Opt. Express 20, A502 (2012).

${ }^{16}$ K. H. Su, Q. H. Wei, X. Zhang, J. J. Mock, D. R. Smith, and S. Schultz, Nano Lett. 3, 1087 (2003).

${ }^{17}$ A. Campion and P. Kambhampati, Chem. Soc. Rev. 27, 241 (1998).

${ }^{18}$ Optical Properties of Metal Clusters, edited by U. Kreibig and M. Vollmer (Springer, Berlin, 1995).

${ }^{19}$ D. E. Aspnes and A. A. Studna, Phys. Rev. B 27, 985 (1983).

${ }^{20}$ F. Bai, M. C. Li, D. D. Song, H. Yu, B. Jiang, and Y. F. Li, J. Solid State Chem. 196, 596 (2012).

${ }^{21}$ T. Fujii, A. David, M. Iza, S. P. DenBaars, E. L. Hu, C. Weisbuch, and S. Nakamura, Phys. Status Solidi C 2, 2836 (2005). 